\title{
La paradoja del octubre rosa
}

\author{
The paradox of pink October
}

Karin Kopitowski

\begin{abstract}
Resumen
En este comentario editorial, la autora repasa la controversia sobre la promoción del rastreo mamográfico del cáncer de mama en el contexto de las campañas de des-información durante el octubre rosa.

Abstract

In this editorial comment, the author reviews the controversy over the promotion of mammographic screening of breast cancer in the context of misinformation campaigns during the pink October.
\end{abstract}

Palabras Clave: Tamizaje Masivo, Neoplasias de la Mama, Servicios Preventivos de Salud, Prevención Secundaria, Prevención Cuaternaria. Keywords: Mass Screening, Breast Neoplasms, Preventive Health Services, Secondary Prevention, Quaternary Prevention.

Kopitowski K. La paradoja del octubre rosa. Evid Actual Pract Ambul. 2019;22(3):e002018.

\section{Información versus desinformación}

Desde que octubre fuera establecido por la Organización Mundial de la Salud (en inglés OMS) como el mes de sensibilización sobre el cáncer de mama ${ }^{1}$, Durante esta época del año las agendas de diversas organizaciones e instituciones públicas y privadas se abarrotan de actividades dirigidas a generar conciencia en la población sobre esta enfermedad. Sin embargo, y a medida que cada año se acrecienta el interés por esta campaña, nos encontramos frente a una creciente problemática que configura un escenario impertinente y contradictorio ${ }^{2}$. La paradoja del octubre rosa consiste en que en un mes en que debiera darse información, el nivel de desinformación es obsceno.

La desinformación es, según la Asociación Española Contra el Cáncer, la provisión de información errónea a través de "titulares alarmistas o morbosos, información científica confusa, creación de estereotipos, invisibilidad de algunos colectivos de enfermos, poco contexto social, uso inapropiado, abuso o frivolización de términos y poca información preventiva” 3 . Omitir información válida y relevante también es desinformación. Y la desinformación, desde este punto de vista, es lo que prima en el octubre rosa.

\section{El mensaje en los medios}

Eslóganes vacíos, falaces. Empresas de todo tipo lucrando con el cáncer descaradamente y sumándose a la desinformación, desde cremas para hacerse el inútil y dañino autoexamen ${ }^{4}$ hasta líneas aéreas que promueven la misma práctica medieval (verFigura $1^{5}$, Figura $2^{6}$ y Figura $3^{7}$ ). Sorprendentemente, los creativos publicitarios transmiten de manera brillante mensajes de espantoso contenido ${ }^{8}$.

Si hablamos de invisibilidad de colectivos, octubre rosa gana por varios cuerpos. ¿Dónde están las mujeres con cáncer avanzado? ¿Dónde las que necesitan (y muchas veces no tienen) cuidados paliativos?

Los discursos rosas sobre el cáncer de mama se caracterizan por la heteronormatividad. Así, las necesidades del colectivo de lesbianas, transexuales, bisexuales, queer e intersexuales (LGT$\mathrm{BI}$ ) en raras ocasiones se ven representadas en las campañas o iniciativas.

\section{Y qué decir de la información ¿científica? que circula}

Veinte años pasaron desde que Gøtzsche y Olsen publicaron en la revista Lancet un artículo cuyo provocador título «Is screening for breast cancer with mammography still justifiable?" (¿Se justifica todavía la detección del cáncer de mama con mamografía?) sacudió el satus quo ${ }^{9}$. ¿Cuánto tiempo más nos vamos a tomar los médicos para leerlo? ¿Cuánto tiempo más nos vamos a tomar para leer toda la evidencia que se publicó después? ${ }^{10-14}$

$Y$ después de leerla ¿cuánto tiempo nos vamos a tomar para contársela a la Sociedad? O haremos como en el pasado con las sangrías, cuando al pobre Pierre Charles Alexandre Louis lo tildaron de loco por demostrar que la práctica era dañina en la década de 1830, y se tardaron más de 100 años en discontinuar su indicación ${ }^{15}$.

No podemos seguir desinformando. No podemos no contar que la mamografía realizada desde los 50 años de edad durante 11 años previene 1 muerte por cáncer de mama por cada 1000 mujeres tamizadas. La mortalidad pasa de 5 en 1000 a 4 en 1000 . Y en las mujeres que la realizan, 4 de cada 1000 reciben un diagnóstico de cáncer que no habría traído problema alguno, por lo tanto son dañadas con el diagnóstico y el tratamiento ${ }^{16}$

¿Hasta cuándo vamos a proponer tamizaje a edades que no fueron incluidas en ningún ensayo clínico? Aún en la edad de "máximo beneficio neto" que es entre los 50 y 69 años, la mamografía realizada cada dos años genera dudas en la comunidad científica en cuanto a su verdadero impacto en la reducción de la mortalidad por cáncer de mama (ver Figura $4{ }^{17,18}$, Figura $5^{19}$ y Figura $\left.6^{20}\right)^{12,14,21}$

¿Hasta cuándo vamos a usar la mentirosa "tasa de sobrevida"para informar los beneficios del tamizaje? El uso de este indicador es inadecuado para las pruebas de cribado de cáncer de mama debido a que se ve distorsionado por diversos sesgos como el de anticipación diagnóstica (cuando el tratamiento de una enfermedad no cambia su pronóstico, la detección temprana en fase asintomática aumenta el tiempo que la persona vive conociendo su diagnóstico en comparación con su detección cuando la patología provoca algún síntoma) o el sesgo de longitud, que se observa cuando el tamizaje aumenta el diagnóstico de formas menos invasivas y de lento crecimiento. Sin embargo, se continúan utilizando las tasas de sobrevida para impresionar más a los lectores sobre los beneficios de esta práctica, independientemente de su reducido y cuestionable efecto sobre la mortalidad ${ }^{22-24}$.

\section{Países con experiencia en el rastreo poblacional de cáncer de mama están abandonando su práctica sis- temática}

En 2014 Asociación Médica Suiza tomó la decisión de desaconsejar formalmente el cribado mamográfico, por su falta de efectividad. En ese país, las campañas generalizadas de tamizaje con mamografía habían evitado una muerte por cáncer de

\footnotetext{
a Servicio de Medicina Familiar y Comunitaria, Hospital Italiano de Buenos Aires. karin.kopitowski@hospitalitaliano.org.ar
} 
mama por cada 1.000 mujeres sometidas a está práctica durante 10 años, sin que esto hubiera afectado a la mortalidad general, ya que esta había sido compensada negativamente por los propios efectos nocivos del cribado (sobrediagnóstico y tratamientos innecesarios) ${ }^{25}$

En octubre de 2016, el Ministro de Salud francés publicó el informe de una investigación independiente sobre mamografía. El informe presentó dos opciones: poner fin al programa nacional de detección de cáncer de mama o bien poner fin al programa actual y poner en marcha un programa radicalmente reformado, que incluyera: a) el suministro de información neutral y completa para las mujeres, el público y los profesionales de la salud; b) el reconocimiento de la controversia científica en la información para las mujeres y los profesionales de la salud; c) la capacitación de los profesionales de la salud para ayudar mejor a las mujeres a tomar decisiones informadas sobre la detección del cáncer de mama; d) el fin de la pesquisa en mujeres de 50 años o menos que están en riesgo promedio; e) la consideración de la detección basada en el riesgo, por lo que las mujeres con bajo riesgo podrían no realizar tamizaje en absoluto, mientras que aquellas en mayor riesgo podrían ser examinadas de manera más intensiva $^{26}$.

Si bien Argentina no dispone de un programa nacional de detección del cáncer de mama, el Instituto Nacional del Cáncer (INC), organismo dependiente de la Secretaría de Gobierno de Salud del Ministerio de Salud y Desarrollo Social de la Nación, recomienda que todas las mujeres entre los 50 y los 69 años de edad, sin antecedentes personales ni familiares de cáncer de mama, deben realizar una mamografía cada uno o dos años ${ }^{27}$. Sin embargo, los principales medios de comunicación difunden mensajes incongruentes con las recomendaciones oficiales ${ }^{28}$, y diversas instituciones de salud, incluso del ámbito público, reali- zan campañas que las contradicen flagrantemente (ver Figura $5^{19}$ y Figura $\left.6^{20}\right)^{29,30}$.

\section{Palabras finales}

Estimados colegas: a quienes ignoran la controversia en torno del tamizaje del cáncer de mama con mamografía, los invito a leer. Tengo muchísima bibliografía disponible.

Para quienes estando al tanto de dicha controversia, difunden información obscenamente falaz, no tengo palabras.

Y hago un llamado para que desde las autoridades de la Confederación Iberoamericana de Medicina Familiar se fije una posición respecto de esta práctica preventiva que esté en sintonía con los principios de la Medicina Familiar (CIMF) y, a su vez, alineada con una política explícita que esta disciplina ya ha adoptado: la prevención Cuaternaria.

Sueño con que se termine este despropósito del octubre rosa, y que éste sea reemplazado por un mes en que los profesionales de la salud recordemos que debemos basar nuestras recomendaciones en la mejor evidencia disponible, comunicar la información de manera balanceada y transparente, y respetar la autonomía de las personas.

Sueño que las mujeres con cáncer no tienen dificultades de acceso a los tratamientos y a cuidados paliativos, y a la cirugía reconstructiva si sufrieron una mastectomía.

Sueño que no hay camiones rosas ofreciendo mamografías sin orden médica y a cualquier edad, sin garantizar el cuidado posterior.

Sueño que ya nadie habla del autoexamen mamario, y que nadie recuerde que alguna vez recomendamos semejante desatino.

Sueño que no falta mucho para eso. Pero apurémonos, llevamos al menos 20 años de atraso.

Figura 1. Video publicitario de una empresa de medicina prepaga. Se atribuye al control anual con mamografía una reducción de la mortalidad del $88 \%$, al sostener que podrían evitarse 15 muertes de cada 17 que ocurren en promedio cada día en Argentina. Fuente: Youtube

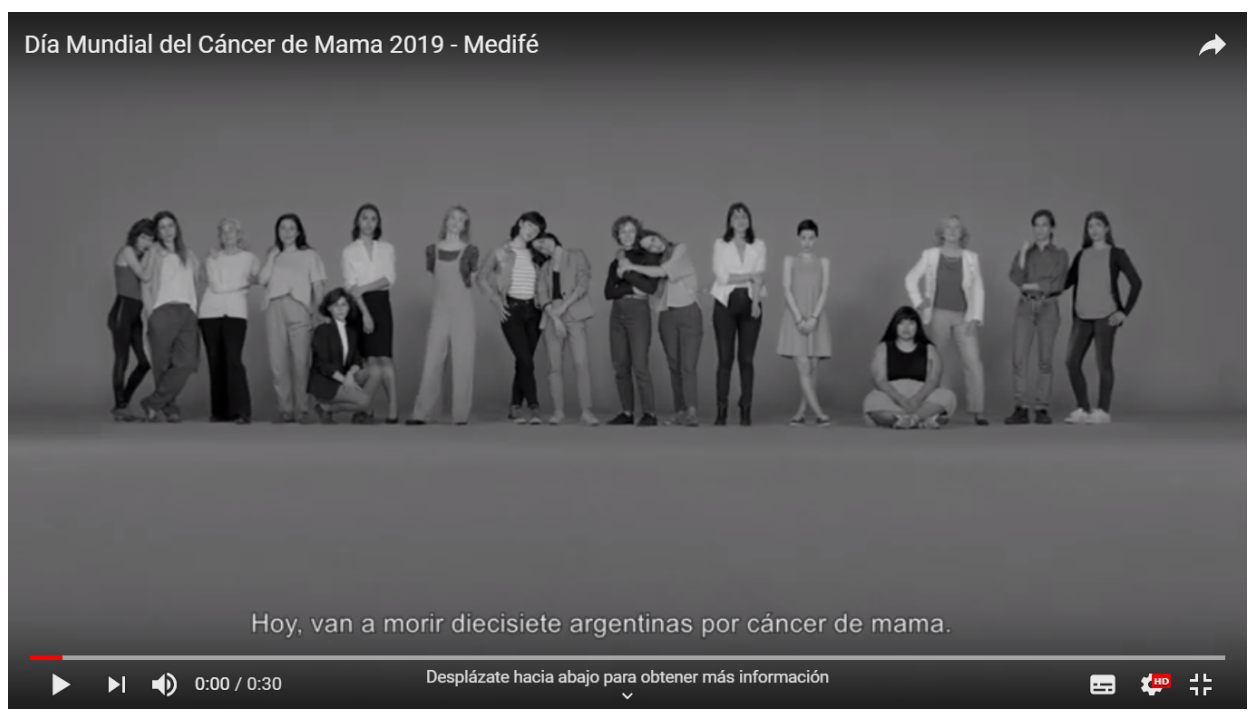


Figura 2. Una conocida marca de cosmética reemplazó las etiquetas de su distintiva crema corporal por una nueva: "Crema para tocarse las Mamas". Fuente: Moda Show

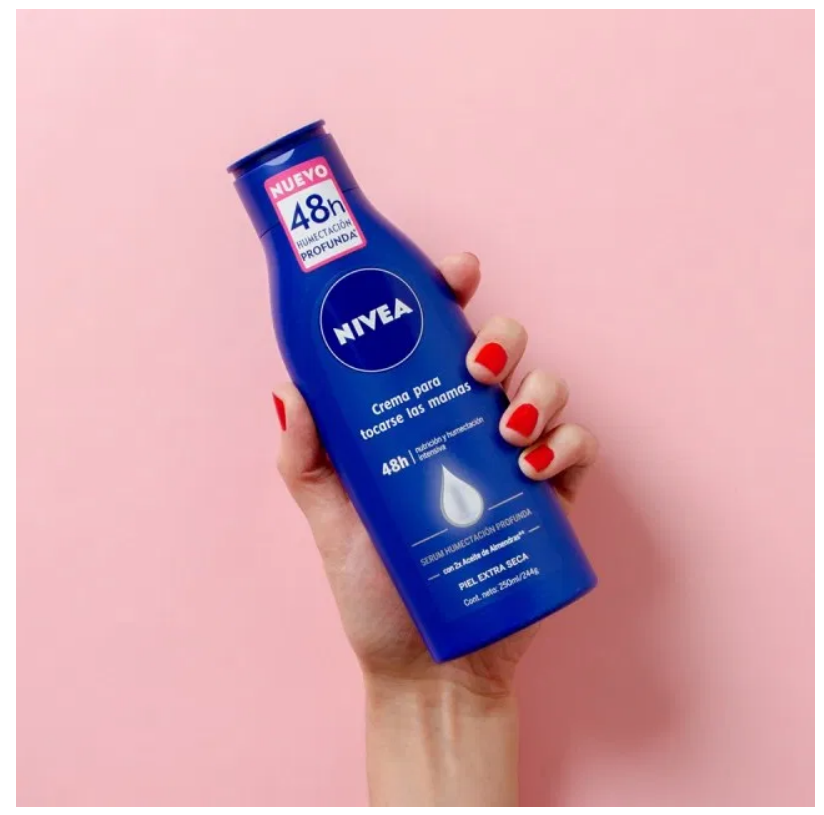

Figura 3. Entre las acciones para alertar sobre la necesidad de prevenir el cáncer de mama, una aerolínea destaca la exhibición de un video que demuestra el modo correcto de hacerse el autoexamen. Fuente: LATAM airlines

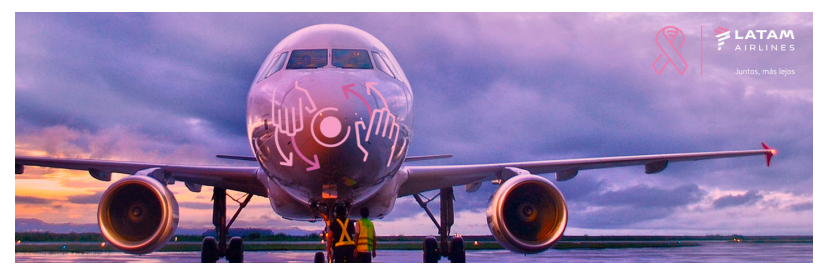

Figura 4. El mamógrafo que recorre el país fue donado por Avón a LALCEC en 1999, y desde ese año realiza mamografías gratuitas en todo el país, a mujeres con edad comprendida entre los 40 y 65 años. Fuente: Radio Mitre

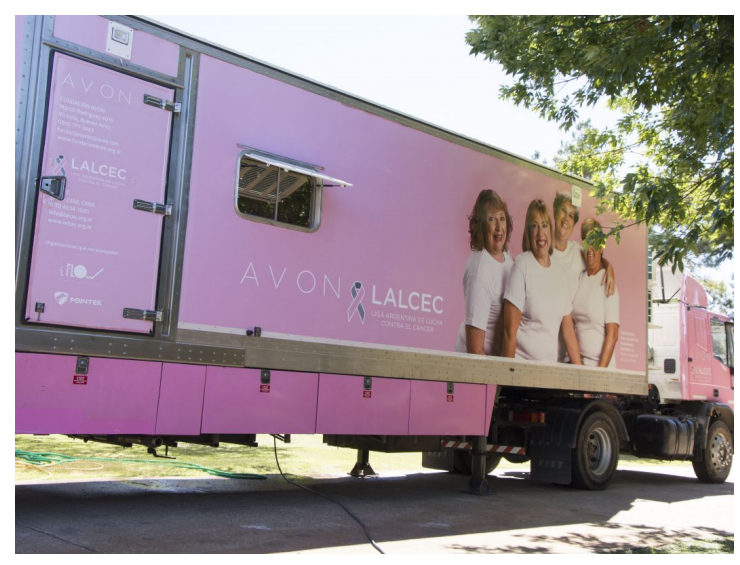

Figura 5. Pieza de difusión de una campaña de prevención del cáncer genito-mamario de un hospital público de la Ciudad de Buenos Aires, que invita a participar a mujeres a partir de los 16 años de edad y propone el uso de ecografía mamaria adicional a la mamografía. Fuente: Facebook

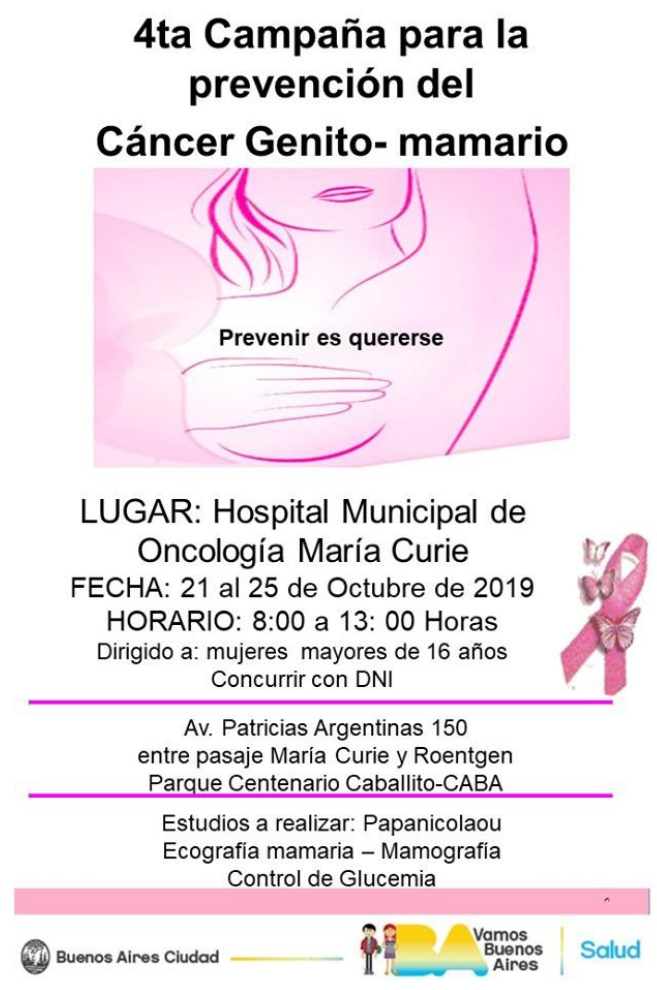

Figura 6. Pieza de difusión de la Universidad de Buenos Aires para la concientización sobre el cáncer mama, que promueve la realización de mamografías antes de la edad recomendada y el autoexamen mamario. Fuente: Instagram

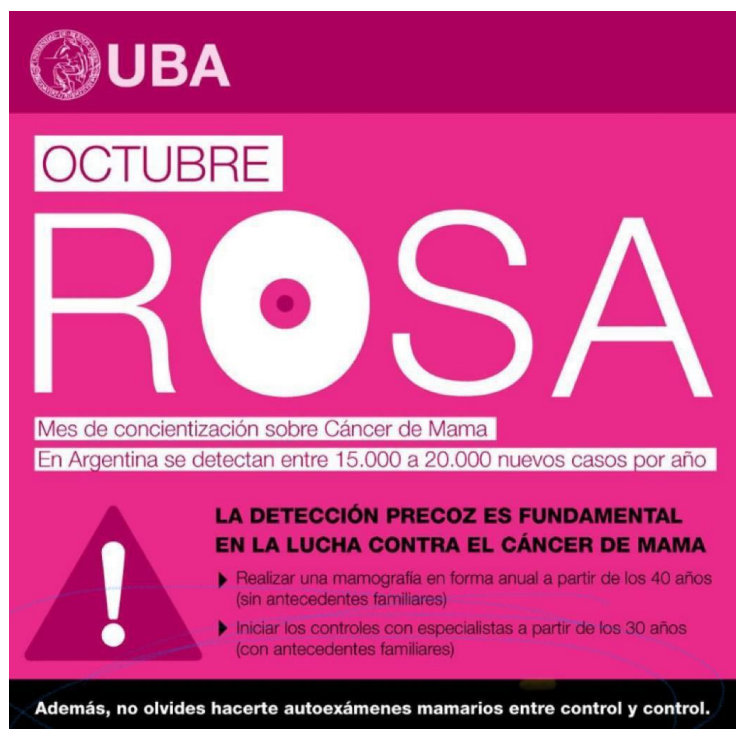




\section{Referencias}

1. Bustreo F, Chestnov O. Una cita importante: octubre es el Mes de Sensibilización sobre el Cáncer de Mama; 2015. Available from: https://www.who. int/es/news-room/commentaries/detail/a-month-to-remember-breast-cancer-awareness-month [Last access: 2019-11-05]

2. Porroche-Escudero A. Problematizando la desinformación en las campañas de concienciación sobre el cáncer de mama. Gaceta Sanitaria. 2017 may;31(3):250-252. Available from: 10.1016/j.gaceta.2016.11.003.

3. AECC. Guía de estilo. Salud y medios de comunicación: cáncer. Madrid, España: Asociación Española Contra el Cáncer; 2006.

4. Kösters JP, Gøtzsche PC. Regular self-examination or clinical examination for early detection of breast cancer. Cochrane Database of Systematic Reviews. 2003;Available from: 10.1002/14651858.CD003373.

5. Medifé. Día Mundial del Cáncer de Mama 2019; 2019. Available from: https://www.youtube.com/watch?v=lg3eyOwQg5M [Last access: 2019-11-05].

6. \#TocateLasMamas con NIVEA; 2019. Available from: https://www.modashow.com.ar/2019/vida-sana/tocatelasmamas-con-nivea/ [Last access: 2019-11-05].

7. Paixão E. Octubre Rosa: las acciones de LATAM en la prevención del cáncer de mama; 2019. Available from: https://www.latam.com/vamos/es_pe/ articulos/en-ruta/octubre-rosa-latam-en-la-prevencion-del-cancer-de-mama/ [Last access: 2019-11-05].

8. Sáez E. Las mejores campañas publicitarias para prevenir el cáncer de mama; 2019. Available from: https://www.enfemenino.com/salud/las-mejorescampanas-publicitarias-para-prevenir-el-cancer-de-mama-s1059316.html [Last access: 2019-11-05].

9. G $\varnothing$ tzsche PC, Olsen O. Is screening for breast cancer with mammography justifiable? Lancet (London, England). 2000 jan;355(9198):129-34. Available from: 10.1016/S0140-6736(99)06065-1.

10. Jørgensen KJ, Gøtzsche PC. Effect of screening mammography on breast cancer incidence. The New England journal of medicine. 2013;368(7):677-8. Available from: 10.1056/NEJMc1215494.

11. Bleyer A, Welch HG. Effect of three decades of screening mammography on breast-cancer incidence. The New England journal of medicine. 2012 nov;367(21):1998-2005. Available from: 10.1056/NEJMoa1206809.

12. Autier P, Boniol M. Mammography screening: A major issue in medicine. European Journal of Cancer. 2018;90:34-62. Available from: 10.1016/j. ejca.2017.11.002.

13. Gøtzsche PC, Jørgensen KJ. Screening for breast cancer with mammography. The Cochrane database of systematic reviews. 2013 jun;(6):001877. Available from: 10.1002/14651858.CD001877.pub5

14. Nelson HD, Cantor A, Humphrey L, Fu R, Pappas M, Daeges M, et al. Evidence Synthesis Number 124 Screening for Breast Cancer: A Systematic Review to Update the 2009 U.S. Preventive Services Task Force Recommendation. Evidence Syntheses. 2016;124(2).

15. Morabia A. Pierre-Charles-Alexandre Louis and the evaluation of bloodletting. Journal of the Royal Society of Medicine. 2006;99:158-160. Available from: 10.1258/jrsm.99.3.158.

16. Harding Center for Risk Literacy Max Planck Institute for Human Development. Early detection of breast cancer by mammography screening;. Available from: https://www.harding-center.mpg.de/en/fact-boxes/early-detection-of-cancer/breast-cancer-early-detection [Last access: 2019-11-05].

17. Realizarán mamografías gratuitas por el Día del Cáncer de Mama; 2019. Available from: https://radiomitre.cienradios.com/realizaran-mamografiasgratuitas-por-el-dia-del-cancer-de-mama/ [Last access: 2019-11-05].

18. Conocé el recorrido 2019 del Mamógrafo Móvil AVON LALCEC; 2019. Available from: https://www.fundacionavon.org.ar/conoce-el-recorrido-2019del-mamografo-movil-avon-lalcec/ [Last access: 2019-11-05].

19. Cáncer de mama: dónde hacerse el control; 2019. Available from: https://www.buenosaires.gob.ar/salud/noticias/cancer-de-mama-donde-hacerseel-control [Last access: 2019-11-05].

20. Octubre rosa; 2019. Available from: http://www.uba.ar/noticia/19647 [Last access: 2019-11-05].

21. U S Preventive Services Task Force. Final Recommendation Statement Breast Cancer: Screening; $2019 . \quad$ Available from: https://www. uspreventiveservicestaskforce.org/Page/Document/RecommendationStatementFinal/breast-cancer-screening1 [Last access: 2019-11-05]

22. Instituto Nacional del Cáncer de los Institutos Nacionales de la Salud de EE UU. Cómo interpretar los números: lo que verdaderamente nos dicen las estadísticas de los exámenes de detección del cáncer; 2018 [Last access: 2019-11-05].

23. Allgood PC, Duffy SW, Kearins O, Sullivan EO, Tappenden N, Wallis MG, et al. Explaining the difference in prognosis between screen-detected and symptomatic breast cancers. British Journal of Cancer. 2011;104(11):1680-1685. Available from: 10.1038/bjc.2011.144.

24. Facciorusso A, Ferrusquía J, Muscatiello N. Lead time bias in estimating survival outcomes. Gut. 2016 mar;65(3):538-9. Available from: 10.1136/ gutjnl-2015-310199.

25. Biller-Andorno N, Jüni P. Abolishing Mammography Screening Programs? A View from the Swiss Medical Board. New England Journal of Medicine. 2014;370(21):1965-1967. Available from: 10.1056/NEJMp1401875.

26. Barratt A, Jørgensen KJ, Autier P. Reform of the national screening mammography program in France. JAMA Internal Medicine. 2018;178:177-178. Available from: 10.1001/jamainternmed.2017.5836.

27. Instituto Nacional del Cáncer Ministerio de Salud y Desarrollo Social. Cáncer de mama;. Available from: https://www.argentina.gob.ar/salud/cancer/ tipos/cancer-de-mama [Last access: 2019-11-05].

28. Spina S, Lombardi V, Terrasa S, Kopitowski K, Villalon G. Cuán precisos son los principales diarios de Argentina al informar sobre los métodos de prevención del cáncer de mama. Rev Argent Salud Pública. 2018;9(37):9-14. Available from: http://rasp.msal.gov.ar/rasp/articulos/volumen37/9-14. pdf.

29. Prensa Ministerio de Salud. Campaña de sensibilización sobre el cáncer de mama: ya se realizaron más de 900 mamografías; 2019. Available from: http://agenciasanluis.com/notas/2019/10/20/campana-de-sensibilizacion-sobre-el-cancer-de-mama-ya-se-realizaron-mas-de-900mamografias/ [Last access: 2019-11-05].

30. Redacción. Se viene una Caminata Rosa para concientizar sobre el cáncer de mama; 2019. //www.diariohuarpe.com/nota/se-viene-una-caminata-rosa-para-concientizar-sobre-el-cancer-de-mama-201910141000?fbclid= IwAR1OJ257NNs2InON89zc9UJ8610oL39smntXK9cGOtclzJM7AZhwhVsFskk [Last access: 2019-11-05]. 